\title{
HER-2 Positive Breast Cancer - a Mini-Review
}

\author{
Hafiz Muhammad Asif ${ }^{1}$, Sabira Sultana ${ }^{1}$, Saeed Ahmed ${ }^{1}$, Naheed Akhtar ${ }^{2}$, \\ Muhammad Tariq3
}

\begin{abstract}
Breast cancer is one of among all cancers with increased incidence, high mortality rate, and high economic and social costs. The the most common type of cancer among females worldwide, breast cancer is actually the uncontrolled proliferation of cells which attain malignancy. Recently it has shown that breast cancer contributes $11 \%$ among all types of cancer diagnosed globally on an annual basis and it is one of the leading causes of death among women. The human epidermal growth factor receptor 2 (HER-2) is a receptor tyrosine-protein kinase erbB-2 normally involved in the proliferation and division of breast cells. In some abnormal cases the HER2 gene does not work correctly and makes too many copies of itself. HER2-positive (HER2+) breast cancers constitute an aggressive type of breast cancer and tend to grow faster and are more likely to spread. However, therapies that specifically target HER2, such as Herceptin ${ }^{\circledR}$ (traztuzumab), are very effective. HER2 targeted therapies, has significantly improved the therapeutic outcome for patients with HER2 positive breast cancer.
\end{abstract}

Keywords: Breast cancer - HER2+ - targeted therapy

Asian Pac J Cancer Prev, 17 (4), 1609-1615

\section{Introduction}

Currently, cancers are leading cause of death all over the world (Saber et al., 2015). Breast cancer is one of among all with increased incidence, high mortality rate, and high economic and social costs (Zainal et al., 2013). It is the most common type of cancer among females worldwide (Cetin et al., 2014).

Breast cancer is actually the uncontrolled proliferation of cells which starts in breast cells and attains malignancy. Recently it has shown that breast cancer contributes $11 \%$ among all types of cancer diagnosed globally annually and it is one of the leading cause of death among women (Majeed et al., 2014). All women regardless of of their racial or ethnic origin or heritage are at risk of breast cancer (Asif et al., 2014). Approximately 1.7 million Women were diagnosed with breast cancer in 2012. It is the most frequently diagnosed cancer among women in 140 of 184 countries worldwide (Erbil et al., 2014). In high income countries, breast cancer is about $30 \%$ cause of cancer deaths and reported to be $29 \%$ of all cancer incidences (Al-Dubai et al., 2012). There were approximately 192,000 new diagnoses of breast cancer and 47,000 deaths in the USA in 2009. Breast cancer is a major public health problem in High Income Countries as well as the Low Income countries (Yusuf et al., 2013). Previous studies revealed that the mortality rate of the breast cancer can be reduced by screening and early detection. Mammography, Breast Self-Examination, and Clinical Breast Examination are some of the methods for screening of the breast cancer (Fouladi et al., 2013). Previous studies have identified a variety of barriers to BC screening, such as poor interactions with doctors and the screening procedure itself (such as pain and discomfort) (Al-Naggar, 2012).

Several risk factors for the development of breast cancer are identified, which include age, menarche, parity, menopausal status, age at first live birth, family history, breast feeding, genetic mutations, (Gürdal et al., 2012) Lifestyle behaviors such as lack of exercise and fatty diet (Berkiten et al., 2012). However, it has long been debated that depression has a role in the development of breast cancer. Depression affects the endocrine and immune function of the body, which may have influence on the initiation of cancer and progression. Women themselves believed that depression also contribute in the development of their breast cancer. However, epidemiology evidences on the relationship between depression and breast cancer incidence are mixed and inconclusive (Hui-Lian Sun et al., 2015).

Clinically, breast cancers are categorized into one of three major subtypes, using the three standard immunohistochemical markers, to facilitate targeted therapy (Qi et al., 2015).

${ }^{1}$ University College of Conventional Medicine, Faculty of Pharmacy \& Alternative Medicine, The Islamia University of Bahawalpur, ${ }^{2}$ Department of Pharmacy, Faculty of Health \& Medical Sciences, The University of Poonch, Rawalakot, AJ\&K, ${ }^{3}$ Margalla Institute of Health Sciences Rawalpindi, Pakistan*For correspondence: drsabirachishti12@gmail.com 
These include 'luminal A' [ER positive and/or progesterone receptor (PR) positive, human epidermal growth factor receptor 2 (HER2) negative], 'luminal B' (ER positive and/or PR positive, HER2 positive), 'HER2 overexpressing' (ER negative, PR negative, HER2 positive), 'basal-like' (ER negative, PR negative, HER2 negative, cytokeratin 5/6 positive and/or epidermal growth factor receptor positive) and 'normal breast-like' tumours (Boyle, 2012). Categorizing breast tumors based on the $\mathrm{ER}, \mathrm{PR}$ and HER/Neu 2 receptor status is necessary in management of breast cancer (Zubeda et al., 2013). Human epidermal growth factor-like receptor 2 (HER2) is a proto-oncogene located on chromosome 17 (Xiu-Juan Li E et al., 2014).

Human epidermal growth factor receptor 2 (HER-2) is a receptor tyrosine-protein kinase erbB-2, also known as CD340 (cluster of differentiation 340), proto-oncogene Neu, Erbb2 (rodent), or ERBB2 (human). It is a protein that in humans is encoded by the ERBB2gene. HER2 belongs to human epidermal growth factor receptor (HER/EGFR/ ERBB). Overexpression of this oncogene has been shown to play an imperative role in the growth and progression of certain violent types of breast cancer. This protein acts as an important biomarker and target of therapy for about $30 \%$ of breast cancer patients (Mitri et al., 2012). These Human epidermal growth factor receptors also include HER1 (which is an EGFR receptor), HER3 (ErbB3), and HER4 (ErbB4) (Bailey et al., 2011). The HER2 protein has a capability of dimerizing with HER1, HER3, or HER4 (Bailey et al., 2011). HER2 has no known ligand, which allows it to always be in open confirmation to dimerize with HER1, HER3, or HER4 (Graus-Porta et al., 1997). Thus, when the HER2 gene overexpressed, it allows for cell growth, survival, and cell differentiation through a signal transduction cascade mediated by the activation of PI3K/Akt and the Ras/Raf/MEK/MAPK pathways (Arteaga et al., 2012). Approximately two millions HER2 proteins present on the surface of HER2-positive cancer cells. These are around 100 times more than a normal cell (Sautter et al., 2009). This HER2 over expression causes cells to grow and proliferate more rapidly. Dimerisation of HER2 is a very important step in the signalling pathway that leads to development of cancer (Lewis et al., 2008).

HER2 is mostly aggressive form of the disease and about one in five women diagnosed with breast cancer worldwide will have HER2-positive breast cancer, (Wolff et al., 2013).

\section{Prognosis of HER 2+ Cancer}

Over expression of the HER2 receptor is generally associated with poor prognosis in patients with breast cancer (Rubin et al., 2001). HER2 expression has also been associated with tumor grade, (Ross et al., 2009; Chibon et al., 2009) positive lymph node metastases (Ross et al., 2009) and mitotic count (Chibon et al., 2009). HER2 positivity may result in increased resistance to endocrine therapy and do not responds non-anthracycline, nontaxane-containing chemotherapy (Wolff et al., 2007). On the contrary, HER2-positive patients give response to anthracycline therapy, as well as to paclitaxel (Albain et al., 1994). Prognostic and predictive value of HER2 status is incessantly evolving. In a recent study, it was shown that increased levels of HER2 gene over expression were associated with poorer outcomes in patients treated with doxorubicin-based therapy in the adjuvant setting (Tubbs et al., 2009). The association of HER2 gene amplification with some breast cancers has allowed for the improvement of agents that specifically target HER2, altering the treatment landscape for these types of cancers. Trastuzumab is a humanized monoclonal antibody to the HER2 protein which was approved for the treatment of metastatic breast cancer in 1998, for the adjuvant treatment of lymph node-positive breast cancer in 2006, and for the adjuvant treatment of lymph node-negative breast cancer in 2008, Lapatinib is a selective inhibitor of the tyrosine kinase activity of HER2 and EGFR. Each of these agents has shown efficacy in patients whose tumors are HER2+ (Ross et al., 2009).

\section{HER +2 Testing}

\section{Immunohistochemistry}

Immunohistochemical staining is the most frequently performed initial test of HER2 status in patients with newly diagnosed invasive breast cancer. Presently, there are 2 commercially available US Food and Drug Administration (FDA)-approved HER2 IHC assays: Dako HercepTest $^{\mathrm{TM}}$ (Dako Corporation, Glostrup, Denmark) and Ventana Pathway ${ }^{\mathrm{TM}}$ (Ventana Medical Systems, Tucson, AZ). Because the HER2 protein is expressed in normal breast epithelial cells, the HER2 IHC assay is a quantitative, rather than a qualitative, test (Wolff et al., 2007).

\section{Fluorescence In Situ Hybridization}

Fluorescence In Situ Hybridization (FISH) can assess gene amplification. In this method fluorescent probe is used to detect the HER2 gene and can also utilize a chromosome 17 probe (CEP17), which acts as an internal control. Currently, there are 3 FDA-approved FISH assays to assess HER2 amplification status. These are PathVysion $^{\mathrm{TM}}$ (Abbott Laboratories, Abbott Park, IL), INFORM (Ventana Medical Systems, Tucson AZ), and PHarmDX (Dako, Glostrup, Denmark) (Sauter et al., 2009).

\section{mRNA by Microarray}

Microarray-based mRNA measurements can be used to assess relative levels of a large number of different mRNA molecules. Various multigene predictor assays exist for use in breast cancer management, including Oncotype $\mathrm{DX}^{\mathrm{TM}}$, Mammaprint ${ }^{\mathrm{TM}}$, and TargetPrint ${ }^{\mathrm{TM}}$. These assays utilize levels of HER2 mRNA, as well as levels of mRNA associated with other genes related to HER2 expression, in determining breast cancer risk or recurrence risk (Ross et al., 2009).

\section{mRNA by RT-PCR}

Reverse transcription polymerase chain reaction (RTPCR) can be used to assess the relative levels of HER2 mRNA. Even though large scale studies have not yet 
been conducted in utilizing this technique to assess HER2 status, but it has significant potential, as it is relatively low-cost and rapid technique (Ross et al., 2009).

Tissue and Serum Enzyme-Linked Immunosorbent Assay

ELISA can be used to determine the concentration of the extracellular domain (ECD) of the HER2 protein. The ECD is separated from the surface of cells by matrix metalloproteases and released into the serum (Lennon et al., 2009). The HER2 ELISA that is commercially available, is the Oncogene Science HER2/neu ELISA (Oncogene Science, Cambridge, MA). The use of ELISA to determine HER2 ECD levels has been approved by the FDA for the monitoring of disease in patients with HER2+ breast cancer (Ross et al., 2009). Some studies have shown a relationship between ECD levels and response to specific therapies. Recently, meta-analysis showed that assessment of HER2 ECD levels may not be informative. A collective analysis of 4 trials showed that baseline ECD levels were not reliably predictive of response to therapy (Lennon et al., 2009). Therefore, more research needs to be carried out in this area before conclusions about the utility of HER2 ECD assessment can be made.

\section{HER2 testing of circulating tumor cells}

To assess HER 2 status by either IHC or FISH, tumor tissue specimens are used. Number of studies has been conducted to investigate the utility of using circulating tumor cells to predict response to therapy, as well as to assess HER2 status without requiring collection of a tissue specimen. Number studies are conducting to assess HER2 status without requiring collection of a tissue specimen but these studies provide interesting data about the use of circulating tumor cells to assess HER2 status in patients with breast cancer, but still there is more research is needed to provide answer.

\section{Target Agents for HER2-Positive Breast Cancer}

\section{Trastuzumab}

Trastuzumab is monoclonal antibody, currently the only approved adjuvant treatment specifically for patients with HER2-positive early stage breast cancer. Its antitumor mechanism of action is unknown but is thought to be mediated by several mechanisms follow $\neg$ ing binding of the antibody to the extracellular domain (ECD) of the HER2 receptor; these mechanisms include antibody-dependent cell-mediated cytotoxicity (ADCC), inhibition of cleavage of the ECD of the HER2 receptor (Molina et al., 2001), inhibition of ligand-independent HER2 receptor dimerization, inhibition of downstream signal transduction pathways, induction of cell-cycle arrest, induction of apoptosis, inhibition of angiogenesis, and interference with DNA repair (Baselga et al., 2001; Spector et al., 2009).

Currently, trastuzumab is recommended by both US (National Comprehensive Cancer Network [NCCN]) and European (St Gallen) guidelines for use as monotherapy after completion of chemotherapy, and in combination with paclitaxel or docetaxel after completion of doxorubicin plus cyclophosphamide, or given concurrently with carboplatin and docetaxel (Goldhirsch et al., 2011; National Comprehensive Cancer Network 2011).

These recom $\neg$ mendations are given on the basis of the results of four large ongoing trials, in addition to number of smaller trials. Results of individual studies that included six randomized clinical trials showed that the combination of trastuzumab with adjuvant chemotherapy produced a significant benefit in disease-free survival (DFS; odds ratio $[\mathrm{OR}]=0.69)$, overall survival $(\mathrm{OR}=$ $0.78)$, locoregional recurrence $(\mathrm{OR}=0.53)$, and distant recurrence $(\mathrm{OR}=0.62)$, as com $\neg$ pared to chemotherapy alone (Yin et al., 2011).

Trastuzumab is also approved and extensively used for patients with metastatic HER2-positive tumor in com $\neg$ bination with paclitaxel 28 or docetaxel (Marty et al., 2005) or as mono $\neg$ therapy (Cobleigh et al., 1999). This strategy was supported by several retrospective trials (Yerushalmi et al., 2010). Furthermore, a randomized phase III trial demonstrated that the combination of trastuzumab with capecitabine resulted in a significant improvement in overall response and time to progression compared with capecitabine alone in patients with HER 2-positive breast cancer who experienced progression during trastuzumab treatment (Von Minckwitz et al., 2009).

Trastuzumab can be administered in combination with a range of other cytotoxic agents, (Wardley et al., 2010; Yardley 2009) including anthracyclines, although simultaneous administration with anthracyclines is associ-ated with an increased risk of cardiotoxicity (Slamon et al., 2001). This risk can be controllable if the cumulative dose of anthracycline is kept low or lesscardiotoxic anthracyclines are used (Untch et al., 2010; Gianni et al., 2010). It is notably, the cardiac dysfunction linked with trastuzumab use, which is thought to be mediated via inhibition of HER2 signaling in cardiac myocytes, seems to be largely reversible (de Azambuja et al., 2009).

\section{Lapatinib}

Lapatinib is another only treatment, than trastuzumab, approved specially for patients with HER2-positive advanced-stage breast cancer. Lapatinib reversibly inhibits the intracellular tyrosine kinase activity of both HER2 and EGFR (also known as HER1), suppressing tyrosine autophosphorylation and thereby downstream pathways, such as the MAPK/Erk1/2 and PI3K/Akt pathways (Spector et al., 2005; Konecny et al., 2006). Preclinical studies showed that lapatinib could inhibit the growth of HER2-positive breast cancer cells that were resistant to trastuzumab (Xia et al., 2004) and that lapatinib could increase the apoptotic effect of anti-HER2 anti-bodies (O'Donovan et al., 2010). These findings proposed that lapatinib might have synergistic activity if com $\neg$ bined with trastuzumab, and that it might have activity in patients with disease resistant to trastuzumab. Central nervous system penetration was also pre $\neg$ dicted, potentially leading to improved control of CNS disease by lapatinib compared with trastuzumab. Early clinical trials indicated superior efficacy modest clinical activity 
(response rates $<10 \%$ ) for single-agent lapatinib in patients whose disease had progressed when giving trastuzumab (Blackwell et al., 2009), but the combination of lapatinib and capecitabine showed significantly efficacy compared to capecitabine alone in such patients (Cameron et al., 2008). A successive randomized trial also showed that the com $\neg$ bination of lapatinib and trastuzumab had better efficacy compared to lapatinib alone in patients whose disease had pro $\neg$ gressed on trastuzumab (Blackwell et al., 2010). Overall the incidence of cardiac toxicity seems to be lower with lapatinib than with trastuzumab, (Perez et al., 2008) possibly owing to different effects on cardiomyocyte mitochon $\neg$ drial ATP stores, (Azim et al., 2009) other differences in the mechanism of action, or to less-sustained inhibition of HER 2 by lapa $\neg$ tinib compared with trastuzumab. Randomized trials have shown that CNS involvement might be reduced by lapatinib administration with chemotherapy (Cameron et al., 2008). The efficacy of lapatinib seems to be confined to patients with great overexpression of HER2, as with trastuzumab, although some trials are still continuing in patients with HER2negative disease (Mayer et al., 2010).

\section{Newer HER2 Drug Therapies}

\section{Pertuzumab}

Pertuzumab is a monoclonal antibody which has the capability of blocking heterodimerization of HER2 with HER3 by interfering with the ligand-dependent HER3 mediated signaling (Gradishar et al., 2012). Pertuzumab drug has only shown modest antitumor clinical efficacy alone; however, it appears to be a very good synergistic drug combined with trastuzumab.

\section{Ttrastuzumab-maytansine [dml]}

Trastuzumab-maytansine [DM1] is an immunoconjugate agent combining trastuzumab with an antimicrotubule cytotoxic chemotherapeutic agent joined together by a covalent bond (Gradishar et al., 2012). It is shown from the Phase I and Phase II studies the drug has well-tolerated and significant objective response rates and improvements of progression-free survival.

\section{Pazopanib}

Pazopanib is a selective multi-targeted receptor tyrosine kinase inhibitor of VEGF receptors $1,2,3$, PDGF receptor $\mathrm{a} / \beta$, and cytokine receptor $\mathrm{c}-\mathrm{kit}$ that inhibits tumor growth and angiogenesis. A phase II study comparing lapatinib (1500 mg) plus placebo and lapatinib (1500 mg) with pazopanib $(800 \mathrm{mg})$ in patients with relapsed HER2 positive inflammatory breast cancer (IBC) was presented by Cristofanilli et al., (2012) at ASCO 2012. 76 patients were examined in a 1:1 double blind study until disease progression, unacceptable toxicity, or death occurred. Due to significantly increased incidence of grades 3 and 4 diarrhea at this dose $(71 \%$ in the combination arm versus $24 \%$ in lapatinib arm) this cohort was closed (Cristofanilli et al., 2012).

Hence, the procedure was revised such that an additional 88 patients were randomized in a 5:5:2 ratio to receive daily monotherapy lapatinib $1,500 \mathrm{mg}$, lapatinib 1,000 mg + pazopanib $400 \mathrm{mg}$, or monotherapy pazopanib $800 \mathrm{mg}$ with a primary endpoint of obtaining an overall response rate (ORR) (Cristofanilli et al., 2012). In this revised cohort, the ORR for patients treated with lapatinib was $47 \%$, lapatinib + pazopanib was $58 \%$, and pazopanib was $31 \%$ (Cristofanilli et al., 2012). The combination exhibited increased toxicity resulting in more dose reductions, modifications, and treatment delays (Cristofanilli et al., 2012). From the study this was came to know that there was no clinical benefit of the combination as opposed to single agent lapatinib (Cristofanilli et al., 2012). In fact, produced increased incidence of toxicities with the combination especially at higher dose of pazopanib.

\section{Afatinib}

Afatinib is an irreversible binder of the HER receptors [HER1, HER2, and HER3] (Gradishar et al., 2012) and possess anti-tumor activity by itself or with chemotherapy in patients with progressive HER2 positive breast cancer. As presented at ASCO 2012, a small phase II trial studying single-agents afatinib (50 mg daily), lapatinib (1500 mg daily) and trastuzumab $(2 \mathrm{mg} / \mathrm{kg}$ weekly after loading dose) as neoadjuvant monotherapy in locally advanced (stage III and inflammatory BC) for 6 weeks pre-operatively suggested that afatinib had higher response rate compared to the other 2 agents $(80 \%$ vs. $75 \%$ vs. $36 \%$ respectively) (Rimawi et al., 2012). However, further studies are needed to search the potential role of afatinib as a single-agent or in combination in HER2 positive breast cancer patients.

\section{Neratinib}

Neratinib is another irreversible binder of the HER receptors [HER1, HER2, and HER3] (Gradishar et al., 2012). This drug has been looked at in studies with patients that are HER2 positive and have been exposed to prior trastuzumab treatment or never have received any anti-HER 2 treatment in the past. In phase II studies, neratinib has been shown to be well-tolerated and shown significant clinical activity (Rabindran et al., 2004). A phase II study looking at 136 HER2 positive patients showed a $24 \%$ response rate in women previously treated with trastuzumab with a progression-free survival at 16 weeks of $59 \%$, and a $56 \%$ response rate in patients who have never received any anti-HER2 treatment prior with a progression-free survival of $78 \%$ at 16 weeks (Burstein et al., 2010). It has also been shown to be more potent and have a more prolonged inhibitory effect as compared to lapatinib and can bypass potential resistance pathways (Burstein et al., 2010). These positive results have led to several phase III studies with neratinib.

\section{Her2-targeted Agents and Cardiotoxicity}

The predominant adverse effects of HER2-targeted agents are the cardiotoxicity. It was came to know in the phase 3 trial of trastuzumab and in successive trials that a significant number of patients who received trastuzumab developed cardiac dysfunction, particularly when it was given along with anthracyclines (Slamon et al., 2001). 
Subsequent studies have shown trastuzumab induced cardiotoxiciry is generally reversible and that trastuzumab may be reintroduced in some patients after resolution of the trastuzumab-induced cardiac dysfunction 51 An FDA review of cardiotoxicity in patients in 4 adjuvant breast cancer trials concluded that there is a 4 - to 6 -fold increase in symptomatic myocardial dysfunction in patients in these trials who were treated with trastuzumab (Fedenko et al., 2009). Generally, patients who are recommended for trastuzumab treatment undergo a baseline assessment of risk for cardiac dysfunction, including a patient history, physical exam, and left ventricular ejection fraction (LVEF) assessment by multiple gated acquisition scan (MUGA) or echocardiogram. It is recommended that trastuzumab be withdrawn from patients with a $\geq 16 \%$ absolute decrease in LVEF from pretreatment values.

\section{Conclusion}

Breast cancer that affects many women worldwide is complex and heterogeneous disease and is divided into many subtypes. Although great studies have been made in the treatment and management of breast cancer and in particular, HER2 positive breast cancer, still the disease has not been conquered. Unlike other subtypes, therapy of HER2+ breast cancer is quite difficult due to absence of a variety of targets. In recent years, many targeted agents take place to combat HER2 positive breast cancer, which provide additional safe and effective treatment options, either as single agents or in combination regimens. Discovery of new targets will lead to develop of new and more effective drugs for the treatment of HER2+ breast cancer. In order to better understand HER2 positive breast cancer, there is a need of further dynamic and innovative clinical trial designs in the form of basic clinical research to improve patient outcomes in drug targeted HER2 positive breast cancer.

\section{References}

Aghdash SA, Ghojazadeh M, Gareh S Sheyklo, et al (2015). Breast Cancer Screening Barriers from the Woman's Perspective: a Meta-synthesis. Asian Pac J Cancer Prev, 16, 3463-71.

Albain KS, Allred DC, Clark GM (1994). Breast cancer outcome and predictors of outcome: are there age differentials? J Natl Cancer Inst Monogr, 16, 35-42.

Al-Dubai SA, Ganasegeran K, Alabsi AM, et al (2012). Exploration of barriers to breast-self examination among urban women in Shah Alam, Malaysia: a cross sectional study. Asian Pac J Cancer Prev, 13, 1627-32.

Al-Naggar RA, Bobryshev YV (2012). Practice and barriers of mammography among Malaysian women in the general population. Asian Pac J Cancer Prev, 13, 3595-600.

Arteaga CL, Sliwkowski MX, Osborne CK, et al (2012). Treatment of HER2-positive breast cancer: current status and future perspectives. Nat Rev Clin Oncol, 9, 16-32.

Asif HM, Sultana S, Akhtar N, et al (2014). Prevalence, Risk Factors and Disease Knowledge of Breast Cancer in Pakistan. Asian Pac J Cancer Prev, 15, 4411-6.

Azim H, Azim HA, Escudier B (2009). Trastuzumab versus lapatinib: the cardiac side of the story. Cancer Treat Rev 35, 633-8.
Bailey TA, Luan H, Clubb RJ, et al (2011). Mechanisms of Trastuzumab resistance in ErbB2-driven breast cancer and newer opportunities to overcome therapy resistance. $J$ Carcinog, 10, 317-28.

Baselga J, Albanell J, Molina MA, et al (2001). Mechanism of action of trastuzumab and scientific update. Semin Oncol, 28, 4-11.

Berkiten A, Sahin NH, Sahin FM, et al (2012). Meta analysis of studies about breast self examination between 2000-2009 in Turkey. Asian Pac J Cancer Prev, 13, 3389-97

Blackwell KL, Burstein HJ, Storniolo AM, et al (2010). Randomized study of lapatinib alone or in combination with trastuzumab in women with ErbB2-positive, trastuzumabrefractory metastatic breast cancer. J Clin Oncol, 28, 1124-30.

Boyle P (2012). Triple-negative breast cancer: epidemiological considerations and recommendations. Ann Oncol, 23, 7-12.

Burstein HJ, Yan Sun Y, Dirix LY, et al (2010). Neratinib, an irreversible ErbB receptor tyrosine kinase inhibitor, in patients with advanced ErbB2-positive breast cancer. J Clin Oncol, 28, 1301-7.

Cameron D, Casey M, Press M, et al (2008). A phase III randomized comparison of lapatinib plus capecitabine versus capecitabine alone in women with advanced breast cancer that has progressed on trastuzumab: updated efficacy and biomarker analyses. Breast Cancer Res Treat, 112, 533-43.

Cetin I, Topcul (2014). M triple negative breast cancer. Asian Pac J Cancer Prev, 15, 2427-31

Chibon F, de Mascarel I, Sierankowski G, et al (2009). Prediction of HER2 gene status in Her2 2+ invasive breast cancer: a study of 108 cases comparing ASCO/CAP and FDA recommendations. Mod Pathol, 22, 403-409.

Cobleigh MA, Vogel CL, Tripathy D et al (1999). Multinational study of the efficacy and safety of humanized anti-HER2 monoclonal antibody in women who have HER2overexpressing metastatic breast cancer that has progressed after chemotherapy for metastatic disease. J Clin Oncol, 17, 2639-48.

Cristofanilli M, Johnston SRD, Manikhas A, et al (2012). A randomized phase II study (VEG108838) of lapatinib plus pazopanib $(\mathrm{L}+\mathrm{P})$ versus lapatinib $(\mathrm{L})$ in patients with ErbB2+ inflammatory breast cancer (IBC). ASCO Meeting Abstracts, 30, 531

De Azambuja, Bedard E, Suter PL, et al (2009). Cardiac toxicity with anti HER 2 therapies: what have we learned so far? Target Oncol, 4, 39-41

Fedenko K, Cortazar P, Keegan P, et al (2009). Trastuzumab cardiotoxicity: FDA review of four adjuvant breast cancer clinical trials leading to trastuzumab marketing approvals [abstract]. J Clin Oncol, 27, 11520.

Floriano-Sanchez E, Rodriguez NC, Bandala C, et al (2014). CYP3A4 expression in breast cancer and its association with risk factors in Mexican women. Asian Pac J Cancer Prev, 15, 3805-9.

Fouladi N, Pourfarzi F, Mazaheri E, et al (2013). Beliefs and behaviors of breast cancer screening in women referring to health care centers in northwest Iran according to the champion health belief model scale. Asian Pac J Cancer Prev, 14, 6857-62

Gianni L, Eiermann W Semiglazov V, et al (2014). Neoadjuvant chemotherapy with trastuzumab followed by adjuvant trastuzumab versus neoadjuvant chemotherapy alone, in patients with HER2-positive locally advanced breast cancer (the NOAH trial): a randomised controlled superiority trial with a parallel HER2-negative cohort. Lancet, 375, 377-84.

Gianni L, Dafni U, Gelber RD, et al (2011). Treatment with trastuzumab for 1 year after adjuvant chemotherapy in 
patients with HER2-positive early breast cancer: a 4 year follow-up of a randomised controlled trial. Lancet Oncol, 12, 236-44.

Goldhirsch A, Wood WC, Coates AS, et al (2011). Strategies for subtypes dealing with the diversity of breast cancer: highlights of the St. gallen international expert consensus on the primary therapy of early breast cancer. Ann Oncol, 22, 1736-47.

Gradishar WJ (2012). HER2 therapy-an abundance of riches. $N$ Engl J Med, 366, 176-8.

Graus-Porta D, Beerli RR, Daly JM, et al (1997). ErbB-2, the preferredheterodimerization partner of all ErbB receptors, is a mediator of lateral signaling. EMBO J, 16, 1647-1655.

Gürdal SO, Saraçoğlu VG, Oran ESO et al (2013). The Effects of Educational Level on Breast Cancer Awareness: ACrossSectional Study in Turkey. Asian Pac J Cancer Prev, 12, 295-300

Hudis CA (2007). Trastuzumab-mechanism of action and use in clinical practice. $N$ Engl J Med, 357, 39-51

Hui-LS, Xiao-Xin Dong, Cong YJ, et al (2015). Depression and the Risk of Breast Cancer: A Meta-Analysis of Cohort Studies. Asian Pac J Cancer Prev, 16, 3233-9.

Konecny GE (2006). Activity of the dual kinase inhibitor lapatinib (GW572016) against HER 2 overexpressing and trastuzumab-treated breast cancer cells. Cancer Res, 66, 42-4.

Lennon S, Barton C, Banken L, et al (2009). Utility of serum HER2 extracellular domain assessment in clinical decision making: pooled analysis of four trials of trastuzumab in metastatic breast cancer. J Clin Oncol, 27, 1685-93.

Lewis Phillips GD, Guangmin Li, Dugger DL, et al (2008). Cancer Res, 68, 9280-90.

Majeed W, Aslam B, Javed I, et al (2014). Breast cancer: major risk factors and recent developments in treatment. Asian Pac J Cancer Prev, 15, 3353-8.

Marty M, Cognetti F, Maraninchi D, et al (2005). Randomized phase II trial of the efficacy and safety of trastuzumab combined with docetaxel in patients with human epidermal growth factor receptor 2 positive metastatic breast cancer administered as first-line treatment: the M77001 study group. J Clin Oncol, 23, 4265-74.

Mayer IA, Arteaga CL (2010). Does lapatinib work against HER2-negative breast cancers? Clin Cancer Res, 16, 1355-7.

Molina MA, Codony-Servat J, Albanell J, et al (2001). Trastuzumab (herceptin), a humanized anti-Her2 receptor monoclonal antibody, inhibits basal and activated Her2 ectodomain cleavage in breast cancer cells. Cancer Res, 61, 4744-9.

National Comprehensive Cancer Network NCCN Practice Guidelines in Oncology. National Comprehensive Cancer Network [online] http://www.ncen.org/professionals/ physician_gls/PDF/breast.pdf (2011).

Erbil N, Bolukbas N (2012) Health beliefs and breast selfexamination among female university nursing students in Turkey. Asian Pac J Cancer Prev, 15, 6525-6529.

O Donovan N, Byrne AT, O Connor AE, et al (2011). Synergistic interaction between trastuzumab and EGFR/HER 2 tyrosine kinase inhibitors in HER 2 positive breast cancer cells. Invest. New Drugs, 29, 752-759.

Perez EA, Koehler M, Byrne J et al (2008). Cardiac safety of lapatinib: pooled analysis of 3689 patients enrolled in clinical trials. Mayo Clin Proc, 83, 679-686.

Qi Yang, Hong-Yan Liu, Dan Liu, et al (2015). Ultrasonographic features of triple-negative breast cancer: a comparison with other breast cancer subtype. Asian Pac J Cancer Prev, 16, 3229-32.

Rabindran SK, Discafani CM, Rosfjord EC (2004). Antitumor activity of HKI-272, an orally active, irreversible inhibitor of the HER-2 tyrosine kinase. Cancer Res, 64, 3958-65.

Rimawi MF, Aleixo SB,Alarcon RA, et al (2012). A neoadjuvant, randomized, open-label phase II trial of afatinib (A) versus trastuzumab $(\mathrm{T})$ versus lapatinib $(\mathrm{L})$ in patients (pts) with locally advanced HER2-positive breast cancer (BC). ASCO Meeting Abstracts, 30, 606.

Ross JS, Fletcher JA(1998). The HER-2/neu oncogene in breast cancer: Prognostic factor, predictive factor, and target for therapy. Oncologist, 3, 237-252.

Rubin I, Yarden Y (2001). The basic biology of HER2. Ann Oncol, 12, 13-8.

Sauter G, Lee J, Bartlett JM, et al (2009). Guidelines for human epidermal growth factor receptor 2 testing: biologic and methodologic considerations. J Clin Oncol, 27, 1323-33.

Slamon DJ, Leyland-Jones B, Shak S, et al (2001). Use of chemotherapy plus a monoclonal antibody against HER2 for metastatic breast cancer that overexpresses HER2. $N$ Engl J Med, 344, 783-92.

Spector NL, Blackwell KL (2009). Understanding the mechanisms behind trastuzumab therapy for human epidermal growth factor receptor 2 positive breast cancer. $J$ Clin Oncol, 27, 5838-47.

Spector NL, Xia W, Burris H, et al (2005). Study of the biologic effects of lapatinib, a reversible inhibitor of ErbB1 and ErbB2 tyrosine kinases, on tumor growth and survival pathways in patients with advanced malignancies. J Clin Oncol, 23, 2502-12.

Tubbs R, Barlow WE, Budd GT, et al (2009). Outcome of patients with early-stage breast cancer treated with doxorubicin-based adjuvant chemotherapy as a function of HER2 and TOP2A status. J Clin Oncol, 27, 3881-3886.

Untch M, Muscholl M, Tjulandin S, et al (2010). First-line trastuzumab plus epirubicin and cyclophosphamide therapy in patients with human epidermal growth factor receptor 2 positive metastatic breast cancer: cardiac safety and efficacy data from the Herceptin, Cyclophosphamide, and Epirubicin (HERCULES) trial. J Clin Oncol, 28, 1473-80.

Von Minckwitz, G du Bois A, Schmidt M, et al (2009). Trastuzumab beyond progression in human epidermal growth factor receptor 2 positive advanced breast cancer: a German Breast Group 26/Breast International Group 03-05 study. J Clin Oncol, 27, 1999-2006.

Wardley AM, Xavier Pivot, Flavia MV, et al (2010). Randomized phase II trial of first-line trastuzumab plus docetaxel and capecitabine compared with trastuzumab plus docetaxel in HER2-positive metastatic breast cancer. J Clin Oncol, 28, 976-83.

Wolff AC, Hammond ME, Hicks DG, et al. (2013). J Clin Oncol, 31, 3997-4013.

Wolff AC, Hammond ME, Schwartz JN, et al (2007). American society of clinical oncology; college of american pathologists. american society of clinical oncology/college of american pathologists guideline recommendations for human epidermal growth factor receptor 2 testing in breast cancer. J Clin Oncol, 25, 118-45.

Xia, W, Liu LH, Ho P, Spector NL (2004). Truncated ErbB2 receptor (p95ErbB2) is regulated by heregulin through heterodimer formation with ErbB3 yet remains sensitive to the dual EGFR/ErbB2 kinase inhibitor GW572016. Oncogene, 23, 646-53.

Xiu-Juan, Zha Q, Xin XY, et al (2014). Lack of Prognostic Value of Human Epidermal Growth Factor-Like Receptor 2 Status in Inflammatory Breast Cancer (IBC): a Meta-analysis. Asian Pac J Cancer Prev, 15, 9615-9.

Yardley DA, Burris HA, Hanson S, et al (2009). Weekly gemcitabine and trastuzumab in the treatment of patients 
with HER2-overexpressing metastatic breast cancer. Clin Breast Cancer, 9, 178-83.

Yerushalmi R, Gelmon K (2010). Treatment beyond progression: is it moving from belief to evidence? Oncologist $\mathbf{1 5}, 796-8$.

Yin W, Jiang Y, Shen Z, et al (2011). Trastuzumab in the adjuvant treatment of HER2-positive early breast cancer patients: a meta-analysis of published randomized controlled trials. PLoS One, 6, 21030.

Yusuf A, Ab Hadi IS, Mahamood Z, et al (2013). Understanding the breast cancer experience: a qualitative study of Malaysian women. Asian Pac J Cancer Prev, 14, 3689-98.

Zainal NZ, Nik-Jaafar NR, Baharudin A, et al (2013). Prevalence of depression in breast cancer survivors: a systematic review of observational studies. Asian Pac J Cancer Prev, 14, 2649-56.

Zubeda S, Kaipa PR, Shaik NA (2013). HER-2/neu status: a neglected marker of prognostication and management of breast cancer patients in India. Asian Pac J Cancer Prev, 14, 2231-5. 